PROCEEDINGS OF THE

AMERICAN MATHEMATICAL SOCIETY

Volume 138, Number 2, February 2010, Pages 405-408

S 0002-9939(09)09860-8

Article electronically published on October 14, 2009

\title{
THE SYMMETRY PRESERVING REMOVAL LEMMA
}

\author{
BALÁZS SZEGEDY
}

(Communicated by Jim Haglund)

\begin{abstract}
In this paper we observe that in the hypergraph removal lemma, the edge removal can be done in such a way that the symmetries of the original hypergraph remain preserved. As an application we prove the following generalization of Szemerédi's Theorem on arithmetic progressions. Let $A$ be an Abelian group with subsets $S_{1}, S_{2}, \ldots, S_{t}$ such that the number of arithmetic progressions $x, x+d, \ldots, x+(t-1) d$ with $x+(i-1) d \in S_{i}$ is $o\left(|A|^{2}\right)$. Then we can shrink each $S_{i}$ by $o(|A|)$ elements such that the new sets don't have any arithmetic progression of the above type.
\end{abstract}

\section{INTRODUCTION}

A directed $k$-uniform hypergraph $H$ on the vertex set $V$ is a subset of $V^{k}$ such that there is no repetition in the $k$ coordinates. The elements of $H$ will be called edges. A homomorphism between two directed $k$-uniform hypergraphs $F$ and $H$ with vertex sets $V(F)$ and $V(H)$ is a map $f: V(F) \mapsto V(H)$ such that $\left(f\left(a_{1}\right), f\left(a_{2}\right), \ldots, f\left(a_{k}\right)\right)$ is in $H$ whenever $\left(a_{1}, a_{2}, \ldots, a_{k}\right)$ is in $F$. The automorphism group $\operatorname{Aut}(H)$ is the group of bijective homomorphisms $\pi: V(H) \mapsto V(H)$. The homomorphism density $t(F, G)$ of $F$ in $G$ is the probability that a map $f: V(G) \mapsto V(H)$ chosen uniformly at random is a homomorphism.

The so-called hypergraph removal lemma (1], 3], 5], 7, , 10])(in the directed setting) says that if the density $t(F, G)$ is small, then a small subset of the edges of $G$ can be removed to make it $F$-free.

Theorem 1 (Removal Lemma). For every $k \in \mathbb{N}, \epsilon>0$ and $k$-uniform directed hypergraph $F$, there is a constant $\delta=\delta(k, \epsilon, F)>0$ such that for every $k$-uniform directed hypergraph $G$ with $t(F, G) \leq \delta$ there is a subset $S \subseteq G$ with $|S| \leq \epsilon|V(G)|^{k}$ such that $t(F, G \backslash S)=0$.

Using this deep result, we observe that the edge removal can be done in a way that the symmetries of $G$ remain preserved. In other words the set $S$ in Theorem 1 can be assumed to be a union of orbits of $\operatorname{Aut}(G)$.

Theorem 2 (Symmetry Preserving Removal Lemma). For every $k \in \mathbb{N}, \epsilon>0$ and $k$-uniform directed hypergraph $F$ there is a constant $\delta_{2}=\delta_{2}(k, \epsilon, F)>0$ such that for every $k$-uniform directed hypergraph $G$ with $t(F, G) \leq \delta_{2}$, there is a subset $S \subseteq G$ with $S \leq \epsilon|V(G)|^{k}$ such that $t(F, G \backslash S)=0$ and furthermore $\operatorname{Aut}(G) \subseteq \operatorname{Aut}(G \backslash S)$.

Received by the editors September 16, 2008, and, in revised form, November 20, 2008.

2000 Mathematics Subject Classification. Primary 05C99.

(C)2009 American Mathematical Society Reverts to public domain 28 years from publication 
Proof. Let $V=V(G)$. Using the original removal lemma it remains to show that if $S \subseteq V^{k}$ satisfies $t(F, G \backslash S)=0$, then there is $S^{\prime} \subseteq V^{k}$ which is $\operatorname{Aut}(G)$ invariant, $t\left(F, G \backslash S^{\prime}\right)=0$ and $S^{\prime} \leq|F||S|$. Such an $S^{\prime}$ is the union of those orbits $O$ of $\operatorname{Aut}(G)$ on $V^{k}$ for which $|O| /|O \cap S| \leq|F|$. Assume that $f: V(F) \mapsto V$ is a homomorphism from $F$ to $G \backslash S^{\prime}$. Then for every fixed $e \in F$ and for a random element $\pi \in \operatorname{Aut}(G)$, the probability is that $\pi(f(e)) \notin G \backslash S^{\prime}$ is less than $1 /|F|$ and so there is some $\pi \in \operatorname{Aut}(\mathrm{G})$ with $\pi(f(F)) \subseteq G \backslash S$, which is a contradiction.

The argument given for the symmetry preserving removal lemma is very general. It applies to various modified versions of the removal lemma. An important such version is the $t$-partite removal lemma, where $t$ is a fixed natural number. The vertex set of a $t$-partite $k$-uniform hypergraph is a $t$-tuple $\left\{V_{i}\right\}_{i=1}^{t}$ of finite sets. An edge of a $t$-partite hypergraph is an element from $\prod_{i=1}^{k} V_{a_{i}}$, where $a_{1}, a_{2}, \ldots, a_{k}$ are $k$ distinct numbers between 1 and $t$. Let $G_{1}, G_{2}$ be two $t$-partite $k$-uniform hypergraphs with vertex sets $\left\{V_{i}\right\}_{i=1}^{t}$ and $\left\{W_{i}\right\}_{i=1}^{t}$. A homomorphism from $G_{1}$ to $G_{2}$ is a $t$-tuple of maps $\left\{\phi_{i}: V_{i} \rightarrow W_{i}\right\}_{i=1}^{t}$ such that $\left(\phi_{a_{1}}\left(r_{1}\right), \phi_{a_{2}}\left(r_{2}\right), \ldots, \phi_{a_{k}}\left(r_{k}\right)\right) \in$ $\prod_{i=1}^{k} W_{a_{i}}$ is an edge in $G_{2}$ whenever $\left(r_{1}, r_{2}, \ldots, r_{k}\right) \in \prod_{i=1}^{k} V_{a_{i}}$ is an edge in $G_{1}$. An automorphism is a bijective homomorphism from $G_{1}$ to $G_{1}$, and the homomorphism density $t\left(G_{1}, G_{2}\right)$ is the probability that a random $t$-tuple of maps $\left\{\phi_{i}: V_{i} \rightarrow W_{i}\right\}_{i=1}^{t}$ is a homomorphism.

We give an example of an application of the symmetry preserving removal lemma, and then we generalize it in the next chapter.

Example 1. Let $S$ be a subset of a group $T$. The Cayley graph $\mathrm{Cy}(T, S) \subseteq T \times T$ is the collection of pairs $(a, b)$ with $a b^{-1} \in S$. The automorphism group of $\mathrm{Cy}(T, S)$ contains $T$ with the action $(a, b) \mapsto(a g, b g)$. Clearly any subset of $T \times T$ invariant under this action of $G$ is a Cayley graph itself. This means that the $T$-orbits of edges in $\mathrm{Cy}(\mathrm{T}, \mathrm{S})$ correspond to elements of $S$.

We apply the symmetry preserving removal lemma to

$$
F=\{(1,2),(1,3),(2,3)\}
$$

with $V(F)=\{1,2,3\}$ and for $G=\mathrm{Cy}(T, S)$. A homomorphism from $F$ to $G$ is a map $f:\{1,2,3\} \mapsto T$ such that $a=f(1) f(2)^{-1}, b=f(2) f(3)^{-1}$ and $c=$ $f(1) f(3)^{-1}$ are all in $S$. Consequently, the number of such homomorphisms is the number

$$
|T||\{(a, b, c) \mid a b=c, a, b, c \in S\}| .
$$

The symmetry preserving removal lemma yields that if $a b=c$ has $o\left(|T|^{2}\right)$ solutions in $S$, then one can remove $o(|T|)$ elements from $S$ such that in the new set there is no solution to $a b=c$. This was first proved by Ben Green [2 for Abelian groups and generalized to groups by Král, Serra and Vena [4].

\section{Cayley hypergraphs}

In this section we describe a potential way of generalizing Cayley graphs to the hypergraph setting and then discuss the symmetry preserving removal lemma on such graphs.

Definition 2.1. Let $G_{1}, G_{2}, \ldots, G_{t}$ be finite groups and let $H$ be a subgroup of $\prod_{i=1}^{t} G_{i}$. The group $H$ acts on each $G_{i}$ by $\left(h_{1}, h_{2}, \ldots, h_{t}\right) g=h_{i} g$, where $\left(h_{1}, h_{2}, \ldots, h_{t}\right) \in H$ and $g \in G_{i}$. A $t$-partite $k$-uniform hypergraph $T$ on the vertex 
set $\left\{G_{i}\right\}_{i=1}^{t}$ is called a Cayley hypergraph if its automorphism group contains $H$ with the previous action.

This definition is very general, so we will start by analyzing a special case. Assume that all the groups $G_{1}, G_{2}, \ldots, G_{t}$ are isomorphic to an Abelian group $A$. Furthermore, to get something interesting we want to assume that $H$ is not too big and not too small. Let $C=\left\{C_{1}, C_{2}, \ldots, C_{r}\right\}$ be a collection of $k$-element subsets of $\{1,2, \ldots, t\}$. Each set $C_{i}$ defines a projection $p_{i}: H \mapsto A^{k}$ to the coordinates in $C_{i}$. Assume that the factor group $A^{C_{i}} / p_{i}(H) \cong A$ and let $\psi_{i}: A^{C_{i}} \rightarrow A$ be a homomorphism with kernel $p_{i}(H)$. Now we pick subsets $S_{i} \subseteq A$ for $1 \leq i \leq r$ and we define the graph $H_{k, t}\left(A,\left\{S_{i}\right\}, C\right)$ as

$$
\bigcup_{i=1}^{r} \psi_{i}^{-1}\left(S_{i}\right)
$$

where $\psi_{i}^{-1}\left(S_{i}\right)$ is the union of cosets in $A^{C_{i}}$ of $p_{i}(H)$ representing an element in $S_{i}$. Note that the way we produced $H_{k, t}\left(A,\left\{S_{i}\right\}, C\right)$ guarantees that its automorphism group contains $H$ as a subgroup.

The symmetry preserving removal lemma for $t$-partite hypergraphs directly implies the following lemma.

Lemma 2.1 (Cayley Hypergraph Removal Lemma). For every $k, t$ natural numbers, $t$-partite $k$-uniform hypergraph $F$ and $\epsilon>0$, there exists a constant $\delta>0$ such that if

$$
t\left(F, H_{k, t}\left(A,\left\{S_{i}\right\}, C\right)\right) \leq \delta,
$$

then there are subsets $S_{i}^{\prime}$ in $A$ of size at most $\epsilon|A|$ such that $t\left(F, H_{k, t}(A\right.$, $\left.\left.\left\{S_{i} \backslash S_{i}^{\prime}\right\}, C\right)\right)=0$.

Example 2. This example uses an idea by Solymosi [8, who showed that the Hypergraph Removal Lemma implies Szemerédi's theorem on arithmetic progressions (even in a multi-dimensional setting). Let $t$ be a natural number, $k=t-1$, and $A$ be an Abelian group. We define $H$ to be the subgroup in $A^{t}$ of the elements $\left(a_{1}, a_{2}, \ldots, a_{t}\right)$ with $\sum_{i=1}^{t} a_{i}=0$ and $\sum_{i=1}^{t}(i-1) a_{i}=0$. Now set

$$
C=\{\{1,2, \ldots, i-1, i+i, \ldots, t\}\}_{i=1}^{t}
$$

and

$$
\psi_{i}\left(a_{1}, a_{2}, \ldots, a_{i-1}, a_{i+1}, \ldots, a_{t}\right)=\sum_{j=1}^{t}(j-i) a_{i} .
$$

The functions $\psi_{i}$ are computed in such a way that $\operatorname{ker}\left(\psi_{i}\right)$ is the projection of $H$ to the coordinates in $C_{i}$.

Let $F$ be the complete $t$-partite $(t-1)$-uniform hypergraph on $t$ points. Lemma 2.1 applied to $F$ and the above hypergraph $H_{t-1, t}\left(A,\left\{S_{i}\right\}, C\right)$ implies that if the system

$$
x_{i}=\sum_{j=1}^{t}(j-i) a_{i} \in S_{i}
$$

has $o\left(|A|^{t}\right)$ solutions, then we can delete $o(|A|)$ elements from each $S_{i}$ such that the previous system has no solution. It is clear that $x_{1}, x_{2}, \ldots, x_{t}$ form a $t$-term arithmetic progression and in fact that any such progression with $x_{i} \in S_{i}$ gives rise to $|A|^{t-2}$ solutions of the previous system. Using this we obtain the following: 
Theorem 3 (Diagonal Szemerédi Theorem). For every $\epsilon>0$ and natural number $t$ there exists $\delta=\delta(\epsilon, t)>0$ such that if $A$ is an Abelian group, $S_{1}, S_{2}, \ldots, S_{t}$ are subsets in $A$ and there are at most $\delta|A|^{2}$ pairs $x, d \in A$ with $x+(i-1) d \in S_{i}(1 \leq$ $i \leq t)$, then we can shrink each $S_{i}$ by at most $\epsilon|A|$ elements such that the new sets don't have such a configuration.

This theorem implies Szemerédi's theorem 9 in the following way. Assume that $S$ is a subset of $A$ not containing any nontrivial $k$-term arithmetic progression. Let us apply Theorem 3 to $S=S_{i}, 1 \leq i \leq t$. We have that if $x+(i-1) d \in S_{i}$ for every $1 \leq i \leq t$, then $d$ is 0 . This means that the condition of Theorem 3 holds with $\delta=|S| /|A|^{2} \leq 1 /|A|$. Now for every $\epsilon>0$, if $|A| \geq 1 / \delta(\epsilon, t)$ we obtain that every $S_{i}$ contains a subset $Q_{i}$ of size at most $\epsilon|A|$ such that the system $\left\{S_{i} \backslash Q_{i}\right\}_{i=1}^{t}$ does not contain any "diagonal arithmetic progression". In particular, $S \backslash \bigcup_{i=1}^{t} Q_{i}$ has to be empty, and so $|S| \leq t \epsilon|A|$.

\section{REFERENCES}

[1] T. Gowers, Quasirandomness, counting and regularity for 3-uniform hypergraphs. Combin. Probab. Comput. 15 (2006), no. 1-2, 143-184. MR2195580(2008b:05175)

[2] B. Green, A Szemerédi type regularity lemma in Abelian groups, with applications, Geom. Funct. Anal. 15 (2005), no. 2, 340-376. MR2153903 (2006e:11029)

[3] Y. Ishigami, A Simple Regularization of Hypergraphs, arXiv:math/0612838v1

[4] D. Král, O. Serra and L. Vena, A combinatorial proof of the removal lemma for groups, J. Combin. Theory Ser. A 116 (2009), 971-978. MR2513645

[5] B. Nagle, V. Rödl and M. Schacht, The counting lemma for regular k-uniform hypergraphs. Random Structures Algorithms 28 (2006), no. 2, 113-179. MR.2198495 (2007d:05084)

[6] V. Rödl and J. Skokan, Regularity lemma for k-uniform hypergraphs. Random Structures Algorithms 25 (2004), no. 1, 1-42 MR2069663 (2005d:05144)

[7] V. Rödl and M. Schacht, Regular partitions of hypergraphs: Regularity lemmas. Combin. Probab. Comput. 16 (2007), no. 6, 833-885. 42. MR2351688 (2008h:05083)

[8] J. Solymosi, A note on a question of Erdös and Graham. Combin. Probab. Comput. 13 (2004), no. 2, 263-267. MR2047239 (2004m:11012)

[9] E. Szemerédi, On sets of integers containing no $k$ elements in arithmetic progression. Acta Arith. 27 (1975), 199-245. MR0369312 (51:5547)

[10] T. Tao, A variant of the hypergraph removal lemma. J. Combin. Theory Ser. A 113 (2006), no. 7, 1257-1280. MR2259060 (2007k:05098)

Department of Mathematics, University of Toronto, 40 St. George Street, Toronto, Ontario, M5S-2E4, Canada

E-mail address: szegedyb@gmail.com 